\title{
Malaria Burden in Pregnancy at Mulago National Referral Hospital in Kampala, Uganda
}

\author{
Fatuma Namusoke, ${ }^{1}$ Niloofar Rasti, ${ }^{2}$ Fred Kironde, ${ }^{3}$ Mats Wahlgren, ${ }^{2}$ and Florence Mirembe ${ }^{1}$ \\ ${ }^{1}$ Department of Obstetrics and Gynaecology, Mulago Hospital, Kampala, P.O. Box 7051, Uganda \\ ${ }^{2}$ Department of Microbiology, Tumor and Cell Biology (MTC), Karolinska Institute, 17177 Stockholm, Sweden \\ ${ }^{3}$ Department of Biochemistry, Makerere University, Kampala, P.O. Box 7072, Uganda
}

Correspondence should be addressed to Mats Wahlgren, mats.wahlgren@ki.se

Received 19 May 2010; Accepted 26 August 2010

Academic Editor: Kwadwo Koram

Copyright (C) 2010 Fatuma Namusoke et al. This is an open access article distributed under the Creative Commons Attribution License, which permits unrestricted use, distribution, and reproduction in any medium, provided the original work is properly cited.

Pregnancy-associated malaria is a major global health concern. To assess the Plasmodium falciparum burden in pregnancy we conducted a cross-sectional study at Mulago Hospital in Kampala, Uganda. Malaria prevalence by each of three measures-peripheral smear, placental smear, and placental histology was 9\% (35/391), 11.3\% (44/389), and 13.9\% (53/382) respectively. Together, smear and histology data yielded an infection rate of 15.5\% (59/380) of active infections and 4.5\% (17/380) of past infections; hence 20\% had been or were infected when giving birth. A crude parity dependency was observed with main burden being concentrated in gravidae 1 through gravidae 3. Twenty-two percent were afflicted by anaemia and $12.2 \%$ delivered low birthweight babies. Active placental infection and anaemia showed strong association $(\mathrm{OR}=2.8)$ whereas parity and placental infection had an interactive effect on mean birthweight $(P=.036)$. Primigravidae with active infection and multigravidae with past infection delivered on average lighter babies. Use of bednet protected significantly against infection $(\mathrm{OR}=0.56)$ whilst increased haemoglobin level protected against low birthweight $(\mathrm{OR}=0.83)$ irrespective of infection status. Albeit a high attendance at antenatal clinics $(96.8 \%)$, there was a poor coverage of insecticide-treated nets (32\%) and intermittent preventive antimalarial treatment (41.5\%).

\section{Introduction}

Malaria is a major public health problem affecting between 300-500 million people annually. Plasmodium falciparum is responsible for the main disease burden afflicting primarily sub-Saharan Africa. In areas with stable malaria transmission, due to protracted exposure to infectious bites, partial protective immunity to clinical malaria is gradually acquired with increasing age. Severe $P$. falciparum malaria is thus predominantly a childhood disease. There is however one exception to this general rule: pregnancy-associated malaria (PAM). Despite their semi-immune status, women become more susceptible to malaria upon pregnancy. In endemic areas, approximately 25 million pregnancies are at risk of $P$. falciparum infection every year, and $25 \%$ of these women have evidence of placental infection at the time of delivery [1-3].

Clinical features of infection during pregnancy vary with the degree of preexistingimmunity and thus the epidemio- logical setting. In high-transmission areas, maternal anaemia and low birthweight (LBW), as a result of prematurity and/or intrauterine growth restriction (IUGR), are the main adverse outcomes of placental infection and tend to be more severe in first pregnancies and in younger mothers [2, 4-8]. These effects are less marked by gravidity in low-transmission areas [9]. Moreover, LBW babies are in general at increased risk of death during infancy. Each year between 100000 to 300000 infant deaths may be attributable to maternal malaria in Africa $[10,11]$.

The pathophysiological processes preceding adverse outcomes in PAM are initiated by the accumulation of $P$. falciparum-infected red blood cells (pRBCs) in placental intervillous spaces, causing inflammatory responses and deposition of fibrinoid material. Adhesive interactions between parasiteencoded erythrocyte surface antigens and intervillous host receptors such as chondroitin sulphate A (CSA), hyaluronic acid (HA), and nonimmune immunoglobulins (Igs) are believed to be involved in the sequestration process [12]. 
The exact details of how sequestration causes LBW are unknown. Local inflammatory immune responses in the infected placenta may induce early labour [13]. IUGR appears to be related to reduced nutrient transport to the foetus due to high parasite and inflammatory cell density [13, 14]. Maternal anaemia may also independently contribute to IUGR, most likely via a reduction in oxygen transport to the foetus [13].

In Uganda, the overall burden of malaria is high and its adverse outcomes to the infected mother and the unborn child are widespread. There is growing awareness that pregnancy-associated malaria is also of importance in areas of low and seasonal transmission worldwide. Although Uganda is regarded as being a malaria-endemic region, the transmission level varies considerably across the country [15]. Similar to studies from other countries, data on malaria burden are mainly available from areas of high transmission. In light of this, we conducted a cross-sectional study to assess the PAM burden in a periurban/urban setting with low, seasonal malaria transmission. Moreover, this is the first study providing baseline data on the burden of PAM and its possible adverse outcomes (anaemia, LBW) at Uganda's National Referral Hospital at Mulago.

\section{Patients and Methods}

2.1. Study Site. Mulago Hospital serves as Uganda's National Referral Hospital and is situated in the capital city of Kampala. In Uganda, there is stable P. falciparum transmission in $95 \%$ of the country. The remaining $5 \%$ of the country, mainly the highland areas with altitudes $>1,600 \mathrm{~m}$, are subject to low and unstable malaria transmission. Kampala is located 1,300-1,500 $\mathrm{m}$ above the sea level close to the equator and experiences a tropical climate with rainfalls throughout the year. The population in the area experiences lowintermediate malaria transmission with the highest peaks toward the end of the two major rainy seasons (March to May and October to December). This study was conducted from October 2004 to January 2005. The rainfall patterns in Kampala were typical, with two peaks, during 2004. There was an average of $146.7 \mathrm{~mm}$ of rainfall between October and December 2004 and $40 \mathrm{~mm}$ in January 2005, a level comparable to the corresponding seasons in previous years. Since the city is built on hills and valleys, the entomological infection rates (EIR) vary considerably depending on the residential/occupational area. Water usually collects in the valley floors resulting in breeding sites for the anopheline mosquitoes. But generally speaking the EIR is low $(<10$ bites per person per year). Except for the main commercial centre, the city and the surrounding areas are essentially rural.

Mulago Hospital has 33,000 antenatal attendances and 23,000 deliveries per year, a maternal mortality ratio of 505 deaths per 100,000 live births, a stillbirth rate of $5 \%$, and an HIV prevalence of about $11 \%$ among pregnant women. The current national policy for prevention of malaria in pregnancy in Uganda is the use of insecticide-treated bednet and intermittent preventive treatment with two doses of sulfadoxine-pyrimethamine. In Uganda, pregnant women are also given iron and folic acid supplementation and antihelminth drugs to prevent anaemia and hookworm infestation, respectively.

\subsection{Study Population and Data Collection. From October} 2004 to January 2005, women delivering at the Mulago Hospital labour suite, aging $\geq 15$ years and $\geq 28$ weeks of gestation, were recruited to the study. Patients with cardiac disease, chronic hypertension, renal disease, clinical AIDS, or diabetes and those with obstetric complications during the present pregnancy, such as preeclampsia, eclampsia, antepartum haemorrhage, and chorioamnionititis were excluded from the study. Full informed consent (or assent for those $<18$ years of age) was obtained from all the participants. On average, five to seven participants were recruited consecutively per day, from 8.00 am to $5.00 \mathrm{pm}$ excluding weekends and public holidays. All ethical aspects of the study were granted by the Makerere University Medical School Research and Ethics Committee and Uganda National Council for Science and Technology (permit No. MV922), and the ethical committee at Karolinska Institutet, Sweden (permit No. 04-533/2). A precoded, standardized questionnaire was used to record pregnancy history, clinical examination outcome, and pregnancy outcome for each study subject. Some key aspects covered included area of residence, age, marital status, occupation, education, parity, visits to antenatal clinic (ANC), bednet use, use of intermittent preventive antimalarial treatment (IPT), iron and folic acid supplementation, gestational age, birth status (live or stillbirth), and birthweight. The information on use of IPT, iron and folic acid supplementation was obtained from interview and/or antenatal card.

2.3. Sample Collection and Laboratory Studies. Venous blood was collected within a few hours (2-4 hours) prior to delivery for peripheral blood diagnosis of malaria and for haemoglobin testing. After delivery, the placentas were collected in $0.9 \% \mathrm{NaCl}$ for smear and histological assessment of malaria. A small incision was made paracentric on the maternal-facing side of the placentas to prepare blood films. Thick and thin blood films of peripheral and placental blood were stained by Giemsa, and malaria diagnosis was assessed by microscopy following standard procedures. A small biopsy of the maternal-facing surface of each collected placenta was also removed and preserved in $10 \%$ neutral buffered formalin. The biopsies were paraffin embedded and stained with haematoxylin and eosin for histological evaluation of placental malaria infection. The slides were examined by a pathologist blinded to other patient data. Reexamination was performed by two different pathologists in all cases where histology and blood films were in disagreement.

2.4. Definitions. Upon histological assessment, placental biopsies were classified according to the following criteria [16, 17]: (a) active acute infection: parasites present in maternal erythrocytes, (b) active chronic infection: presence of parasites and a significant amount of pigment deposition in fibrin or monocytes within fibrin, (c) past infection: 
presence of pigment within fibrin only, no parasites, and (d) not infected: no evidence of parasites or pigment. Low birthweight is defined as weight $<2500 \mathrm{~g}$. Anaemia is defined as haemoglobin $(\mathrm{Hb})$ level $<11 \mathrm{~g} / \mathrm{dl}$ and severe anaemia as $\mathrm{Hb}<7 \mathrm{~g} / \mathrm{dl}$. Preterm delivery is regarded as deliveries occurring prior to 37 weeks of gestation. Bednet users refer to individuals using net of any category (untreated as well as insecticide-treated nets). ITN users refer to those using insecticide-treated nets only.

2.5. Statistical Analysis. Data were entered and verified by two independent individuals using Epi Info version 6.1 and exported to SPSS version 12.0 for further analysis. Placental $P$. falciparum infection, anaemia, and birthweight were regarded as principal outcomes. Potential risk factors or confounders considered were area of residence, literacy, age, use of bednet, IPT, and iron and folic acid supplementation. Following the assessments of the crude effect of each risk factor, the list of interesting risk factors was organised into three blocks: background, intermediate and proximate risk factors. Background risk factors were adjusted for each other: intermediate for background and intermediate, and proximate for all other risk factors. Adjusted odds ratios were obtained using logistic regression. The significance of each variable was reconsidered by backward stepwise elimination. Variables with a $P$-value of $<.1$ were included in the final model. Due to the presence of missing values in the dataset, missing value analysis was performed prior to logistic regression modelling in order to assess the total number and randomness of missing values.

Proportions were compared using $\chi^{2}$ tests and validated at a 5\% significance level. Multiway univariate analysis of variance (ANOVA) was used to study the effects of placental infection, gravidity and age, and combinations thereof, on haemoglobin level and birthweight.

\section{Results}

3.1. General Description. A total of 399 women who consented to take part in the study were recruited between October 2004 and January 2005. The age of the participants ranged from 15 to 44 years, median 20 (IQR: 1825). Most participants were residents of Kampala (68.6\%) and Wakiso $(22.9 \%)$ districts. The majority had primary $(46.4 \%)$ or secondary $(42.3 \%)$ level of education; $66.3 \%$ were housewives or unemployed; $74 \%$ were married. Most women $(96.8 \%)$ had attended an antenatal clinic at least once during the present pregnancy. One hundred and sixty five $(41.5 \%)$ had received intermittent preventive antimalarial treatment (IPT); of these, the majority had received one dose (74.5\%), whereas $20.5 \%$ had received the two recommended doses. Sulfadoxine-pyrimethamine (SP) was the drug of choice $(89.3 \%)$. Other preventive measures taken during pregnancy consisted of the use of mosquito bednets: two thirds $(267 / 397 ; 67.3 \%)$ utilized nets of any sort: $32 \%$ (127/397) were strict insecticide-treated net (ITN) users. Most women had also received iron (79.3\%) and folic acid (70.4\%) supplementation during the present pregnancy (see
Table 1 for general characteristics). The study population consisted of 196 (49.4\%) gravidae 1 (G1) or primigravidae, $142(35.8 \%)$ gravidae 2-3 (G2-3), and 59 (14.9\%) gravidae 4 or above $(\geq \mathrm{G} 4)$. A number of factors showed crude associations with gravidity. Primigravidae tended to be younger $(P<.0001)$ and more literate $(P=.001)$, delivered more low-birthweight babies $(P=.06)$, used less IPT $(P<.0001)$, and were more afflicted by placental malaria infection $(P=$ .035 ) as compared to multigravidae (G2 and above; Table 2).

3.2. Prevalence of Malaria. The prevalence of malaria by each of the three measures peripheral smear, placental smear and placental histology, was 9\% (35/391), 11.3\% (44/389) and $13.9 \%$ (53/382), respectively. Out of 53 women with histological evidence of infection, 34 (64.1\%) were classified as acute, $2(3.8 \%)$ as chronic, and $17(32.1 \%)$ as past infection. P. falciparum was the sole species found in all cases. A total of 380 cases, where placental histology and the corresponding blood film data were available, were used for further associative analysis. In order to avoid loss of data, all cases diagnosed as being acute or chronic infection by histology or as $P$. falciparum positive by placental blood film examination were regarded as active infection. Based on the new case definition criteria, a total of $15.5 \%(59 / 380)$ and $4.5 \%(17 / 380)$ had active versus past placental infection, respectively. Peripheral parasites were present in 50.9\% $(28 / 55)$ and $5.9 \%(1 / 17)$ of cases with active versus past infection. In patients with no evidence of active or past placental infection, only $1.3 \%(4 / 304)$ had peripheral parasitaemia. Placental infection could thus be used as a reliable measure of malaria burden in the remainder of the analysis.

3.3. Prevalence of Anaemia and Low Birthweight. Twenty-two percent of the women were anaemic $(\mathrm{Hb}<11 \mathrm{~g} / \mathrm{dl})$ prior to delivery. Severe anaemia $(\mathrm{Hb}<7 \mathrm{~g} / \mathrm{dl})$ was however uncommon $(3 / 389 ; 0.8 \%)$. The mean haemoglobin level was $12.3 \mathrm{~g} / \mathrm{dl}(\mathrm{IQR}=10.9-13.4)$ (Table 1$)$. The overall prevalence of stillbirths was $2.8 \%(11 / 389)$ and preterm deliveries $3.1 \%$ (12/389). Among live-born babies, 12.2\% (46/378) were of low birthweight and the mean birthweight was $3100 \mathrm{~g}$ (IQR $=2800-3500)$ (Table 1$)$.

3.4. Risk Factors Associated with Placental Malaria. Table 3 illustrates crude and adjusted odds ratios for factors associated with placental $P$. falciparum infection. Whilst higher age and use of bednet were found protective, district of residence, educational level, and use of IPT showed no association. Being multigravid ( $\geq \mathrm{G} 4$ ) was protective in the crude analysis $(\mathrm{OR}=0.38 ; \mathrm{CI}=0.14-0.88 ; P<.05)$ (Figure $1(\mathrm{a}))$; the effect was, however, lost in the adjusted model (Table 3 ). As gravidity and age are highly correlated variables, an agestratified analysis was performed to separate out the effect of the two. Gravidity groups were stratified by two age groups (15-19 years versus $\geq 20$ years). A trend of higher infection rate, although not significant, was observed in young primigravidae (G1: $33 / 126=23.1 \%$ versus $\geq G 2: 4 / 28$ $=14.3 \% ; \mathrm{OR}=2.1 ; \mathrm{CI}=0.69-6.6 ; P=.18)$. The analysis was, 
TABLE 1: General characteristics.

\begin{tabular}{|c|c|}
\hline \multicolumn{2}{|l|}{ District (\%) } \\
\hline Kampala & 68.6 \\
\hline Wakiso & 22.9 \\
\hline Other & 8.5 \\
\hline \multicolumn{2}{|l|}{ Ethnic group (\%) } \\
\hline Ganda & 62.8 \\
\hline Nyankole & 8.0 \\
\hline Soga & 6.0 \\
\hline Rwandese & 5.3 \\
\hline Other & 17.9 \\
\hline Median age (years) & 20 (IQR: 18-25) \\
\hline \multicolumn{2}{|l|}{ Education (\%) } \\
\hline Illiterate & 6.9 \\
\hline Primary & 46.4 \\
\hline Secondary & 42.3 \\
\hline Higher & 4.3 \\
\hline \multicolumn{2}{|l|}{ Marital status (\%) } \\
\hline Married & 74.0 \\
\hline Single & 20.4 \\
\hline Cohabitant & 5.6 \\
\hline \multicolumn{2}{|l|}{ Occupation (\%) } \\
\hline Housewife & 55.8 \\
\hline Peasant & 2.9 \\
\hline Student & 9.7 \\
\hline Casual worker & 18.2 \\
\hline Professional job & 2.9 \\
\hline Unemployed & 10.5 \\
\hline Visit to ANC (\%) & 96.8 \\
\hline Received IPT (\%) & 41.5 \\
\hline Use of bednet (\%) & 67.3 \\
\hline Use of ITN (\%) & 32.0 \\
\hline Median Hb level (g/dl) & 12.3 (IQR: 10.9-13.4) \\
\hline $\mathrm{Hb}<11 \mathrm{~g} / \mathrm{dl}(\%)$ & 22.0 \\
\hline Folic acid supplementation (\%) & 70.4 \\
\hline Iron supplementation (\%) & 79.3 \\
\hline Median birthweight (g) & 3100 (IQR: 2800-3500) \\
\hline LBW (\%) & 12.2 \\
\hline Preterm delivery (\%) & 3.1 \\
\hline Stillbirth (\%) & 2.8 \\
\hline Caesarean (\%) & 17.6 \\
\hline Peripheral malaria (\%) & 9.0 \\
\hline \multicolumn{2}{|l|}{ Placental malaria (\%) } \\
\hline Histology & 13.9 \\
\hline Blood smear & 11.3 \\
\hline
\end{tabular}

ANC: antenatal clinic, at least one visit during the present pregnancy. IPT: intermittent preventive antimalarial treatment, at least one dose. ITN: insecticide-treated net. LBW: low birthweight. however, limited by the absence of higher parities $(\geq \mathrm{G} 4)$ in the younger age group.

3.5. Risk Factors Associated with Anaemia. The crude analysis and the adjusted final model, both, identified a strong association between active placental malaria infection and anaemia $(\mathrm{OR}=2.76 ; \mathrm{CI}=1.4-5.5 ; P=.003)($ Table 4 , Figure 1(b)). The mean haemoglobin level was $11.6 \mathrm{~g} / \mathrm{dl}$ (CI: $11.1-12.3$ ) versus $12.6 \mathrm{~g} / \mathrm{dl}$ (CI: 12.3-12.9) in patients with active infection or no infection, respectively, $(P=.02)$. No associations were observed with any of the other considered risk factors. Of note, use of bednet showed a trend towards a protective effect (Table 4 ).

3.6. RiskFactors Associated with Low Birthweight. Factors associated with low birthweight are outlined in Table 5. Increased haemoglobin level and to some extent age showed protective association with low birthweight. Increased gravidity ( $\geq$ G4) showed a crude but not adjusted protective association. No overall associations were found with district of residence, education, placental malaria infection, use of bednet, IPT, and iron and folic acid supplementation. A significant association was, however, found when interactions between gravidity and placenta infection groups and their effect on mean birthweight were analyzed (Table 6; $P=$.036). However, interpretation of the effect size of gravidity and placental infection on mean birthweight becomes complicated in light of the significant covariation of gravidity by age $(P=.034$; see Table 6 and Supplementary table in supplementary material available online at doi: $10.406 / 2010 / 913857)$. To analyze the pattern of the identified interaction, the age parameter was thus fixed at a mean value in all subgroups (Tables 7 and 8). The birthweight was on average lower in primigravidae irrespective of infection status. The smallest mean birthweight in primigravidae were found in mothers with active infection, whereas in multigravidae it was observed in mothers with past infection (Tables 7 and 8).

\section{Discussion}

Data on the burden of malaria in pregnancy, in particular from areas of low transmission, are scarce in Uganda as well as in other countries. A cross-sectional study was thus conducted from October 2004 to January 2005 in the periurban/urban setting of Kampala where the population experiences relatively low and seasonal malaria transmission. Exploiting placenta histology and blood film examination, a high proportion of the study population was found burdened with active placental infection (15.5\% in total). In addition, histological examination identified a number of women with past placental infection (4.5\%). Adverse clinical outcomes associated with malaria in pregnancy are linked to pathological changes in the parasite-burdened placentas [18], and histology is the only method that provides insight on pathological changes as well as timing of infection (acute, chronic, and past). Past infections would have been missed had we relied solely on blood film examination. 
TABLE 2: General characteristics by gravidity.

\begin{tabular}{|c|c|c|c|c|c|}
\hline \multirow[t]{2}{*}{ Characteristics } & \multirow{2}{*}{$\begin{array}{l}\text { Primigravidae (\%) } \\
\text { G1 (n 196) }\end{array}$} & \multicolumn{3}{|c|}{ Multigravidae (\%) } & \multirow[t]{2}{*}{$P$-value for gravidity change $\mathrm{e}^{\mathrm{a}}$} \\
\hline & & $\mathrm{G} 2-3(n 142)$ & & $\geq \mathrm{G} 4(n$ 59) & \\
\hline \multicolumn{6}{|l|}{ District of residence } \\
\hline Kampala & 70.2 & 67.4 & & 66.7 & .97 \\
\hline Wakiso & 22.0 & 23.2 & & 25.0 & \\
\hline Other & 7.9 & 9.4 & & 8.3 & \\
\hline \multicolumn{6}{|l|}{ Age (years) } \\
\hline $15-19$ & 68.9 & & 14.9 & & $<.0001$ \\
\hline$\geq 20$ & 31.1 & & 85.1 & & \\
\hline \multicolumn{6}{|l|}{ Education } \\
\hline Illiterate & 4.4 & 7.1 & & 12.5 & .001 \\
\hline Primary & 38.3 & 52.0 & & 60.7 & \\
\hline Higher education & 57.2 & 40.9 & & 26.8 & \\
\hline Visit to ANC & 97.2 & 95.6 & & 98.2 & .61 \\
\hline Recieved IPT & 31.6 & 49.3 & & 55.7 & $<.0001$ \\
\hline Bednet (of any kind) & 65.8 & 65.9 & & 75.0 & .38 \\
\hline Stillbirth & 3.7 & & 2.0 & & .31 \\
\hline LBW (<2500 g) & 15.2 & & 9.0 & & .06 \\
\hline Anaemia $(<11 \mathrm{~g} / \mathrm{dL})$ & 23.2 & 22.6 & & 18.3 & .73 \\
\hline \multicolumn{6}{|l|}{ Placental malaria } \\
\hline Active infection & 19.6 & 12.5 & & 10.3 & .035 \\
\hline Past infection & 3.8 & 7.4 & & 0 & \\
\hline
\end{tabular}

ANC: antenatal clinic, at least one visit during the present pregnancy.

IPT: intermittent preventive antimalarial treatment, at least one dose.

${ }^{a}$ Chi-square test used. Age, stillbirth, and LBW were analysed using multigravidae as a pooled group ( $\left.\geq \mathrm{G} 2\right)$.

TABLE 3: Risk factors associated with placental malaria.

\begin{tabular}{|c|c|c|c|c|c|}
\hline Factor level & Risk factor & Crude OR & Adjusted OR & $(95 \% \mathrm{CI})$ & $P$-value \\
\hline \multirow[t]{6}{*}{ Background } & Age (continuous per year) & $0.95^{*}$ & 0.95 & $(0.89-1.00)$ & .066 \\
\hline & District of residence & & & & \\
\hline & Kampala & 1.0 & 1.0 & & \\
\hline & Wakiso & 1.18 & 1.4 & $(0.74-2.60)$ & .31 \\
\hline & Other & 1.80 & 1.9 & $(0.79-4.74)$ & .15 \\
\hline & Education (continuous per level) & 0.79 & 0.75 & $(0.48-1.17)$ & .21 \\
\hline \multirow[t]{4}{*}{ Intermediate } & Gravidity & & & & \\
\hline & G1 & 1.0 & 1.0 & & \\
\hline & G2-3 & 0.81 & 1.0 & $(0.54-2.07)$ & .88 \\
\hline & $\geq \mathrm{G} 4$ & $0.38^{*}$ & 0.72 & $(0.21-2.50)$ & .61 \\
\hline \multirow[t]{5}{*}{ Proximate } & Received IPT & & & & \\
\hline & None & 1.0 & 1.0 & & \\
\hline & 1 dose of SP & 0.93 & 1.11 & $(0.60-2.07)$ & .73 \\
\hline & 2 doses of SP & 0.55 & 0.49 & $(0.16-1.49)$ & .21 \\
\hline & Used bednet & $0.56^{*}$ & 0.56 & $(0.31-0.99)$ & .046 \\
\hline
\end{tabular}

* Significant associations $(P<.05)$.

Of note, a minor proportion of women with no evidence of placental infection (1.3\%) were afflicted by peripheral parasites. Individuals in endemic areas can, however, harbor circulating parasites asymptomatically. Hence, the mere presence of peripheral parasites coinciding with pregnancy is not a proof of their involvement in placental sequestration and adverse clinical outcomes. Placental infection was thus used as a reliable measure of PAM burden in this study.

The majority of the placental malaria cases was concentrated among gravidae 1 through 3 . Observed pattern may be explained by the development of protective antibodies by successive pregnancies. Acquired antibodies recognize 
TABLE 4: Risk factors associated with anaemia $(<11 \mathrm{~g} / \mathrm{dl})$.

\begin{tabular}{|c|c|c|c|c|c|}
\hline Factor level & Risk factor & Crude OR & Adjusted OR & $(95 \% \mathrm{CI})$ & $P$-value \\
\hline \multirow[t]{6}{*}{ Background } & Age (continuous per year) & 1.0 & 0.98 & $(0.93-1.04)$ & .51 \\
\hline & District of residence & & & & \\
\hline & Kampala & 1.0 & 1.0 & & \\
\hline & Wakiso & 1.15 & 1.11 & $(0.60-2.07)$ & .74 \\
\hline & Other & 0.40 & 0.44 & $(0.13-1.52)$ & .20 \\
\hline & Education (continuous per level) & 1.14 & 1.14 & $(0.73-1.77)$ & .57 \\
\hline \multirow[t]{7}{*}{ Intermediate } & Gravidity & & & & \\
\hline & G1 & 1.0 & 1.0 & & \\
\hline & $\mathrm{G} 2-3$ & 1.04 & 1.0 & $(0.56-1.80)$ & .98 \\
\hline & $\geq \mathrm{G} 4$ & 0.86 & 0.70 & $(0.30-1.63)$ & .51 \\
\hline & Placental malaria (active + past $)$ & $2.31^{*}$ & 2.35 & $(1.27-4.35)$ & .006 \\
\hline & Active infection & $2.66^{*}$ & 2.76 & $(1.40-5.45)$ & .003 \\
\hline & Past infection & 1.37 & 1.53 & $(0.44-4.66)$ & .48 \\
\hline \multirow[t]{7}{*}{ Proximate } & Used bednet & 0.73 & 0.67 & $(0.37-1.20)$ & .18 \\
\hline & Received IPT & & & & \\
\hline & None & 1.0 & 1.0 & & \\
\hline & 1 dose of SP & 1.05 & 1.17 & $(0.64-2.13)$ & .62 \\
\hline & 2 doses of SP & 1.46 & 1.17 & $(0.47-2.86)$ & .74 \\
\hline & Iron supplementation & 1.51 & 1.96 & $(0.67-5.71)$ & .22 \\
\hline & Folic acid supplementation & 1.26 & 0.70 & $(0.29-1.71)$ & .43 \\
\hline
\end{tabular}

* Significant associations $(P<.05)$.

TABLE 5: Risk factors associated with low birthweight $(<2500 \mathrm{~g})$.

\begin{tabular}{|c|c|c|c|c|c|}
\hline Factor level & Risk factor & Crude OR & Adjusted OR & $(95 \% \mathrm{CI})$ & $P$-value \\
\hline \multirow[t]{6}{*}{ Background } & Age (continuous per year) & $0.91^{*}$ & 0.93 & $(0.86-1.0)$ & .068 \\
\hline & District of residence & & & & \\
\hline & Kampala & 1.0 & 1.0 & & \\
\hline & Wakiso & 0.86 & 0.98 & $(0.45-2.13)$ & .97 \\
\hline & Other & 0.21 & 0.21 & $(0.01-1.06)$ & .14 \\
\hline & Education (continuous per level) & 1.1 & 1.05 & $(0.61-1.81)$ & .87 \\
\hline \multirow[t]{8}{*}{ Intermediate } & Gravidity & & & & \\
\hline & G1 & 1.0 & 1.0 & & \\
\hline & G2-3 & 0.69 & 1.22 & $(0.54-2.77)$ & .63 \\
\hline & $\geq \mathrm{G} 4$ & $0.30^{*}$ & 0.70 & $(0.14-3.51)$ & .67 \\
\hline & Placental malaria (active + past) & 1.48 & 0.72 & $(0.29-1.80)$ & .48 \\
\hline & Active infection & 1.61 & 0.69 & $(0.24-1.96)$ & .49 \\
\hline & Past infection & 1.05 & 0.77 & $(0.16-3.77)$ & .75 \\
\hline & Haemoglobin level & $0.83^{*}$ & 0.83 & $(0.70-0.99)$ & .033 \\
\hline \multirow[t]{7}{*}{ Proximate } & Use of bednet & 1.33 & 1.65 & $(0.72-3.79)$ & .24 \\
\hline & Received IPT & & & & \\
\hline & None & 1.0 & 1.0 & & \\
\hline & 1 dose of SP & 1.20 & 1.21 & $(0.56-2.63)$ & .63 \\
\hline & 2 doses of SP & 0.84 & 0.96 & $(0.30-3.09)$ & .94 \\
\hline & Iron supplementation & 0.74 & 0.19 & $(0.02-1.58)$ & .12 \\
\hline & Folic acid supplementation & 1.0 & 4.48 & $(0.57-34.9)$ & .15 \\
\hline
\end{tabular}

* Significant associations $(P<.05)$. 
TABLE 6: Multiway ANOVA—birthweight as dependent variable.

\begin{tabular}{lcccccc}
\hline & Type III sum of squares & Df & Mean square & $F$ & $P$-value & Partial Eta Squared \\
\hline Corrected model & $9.063 \mathrm{E6}$ & 7 & 1294773.8 & 4.45 & .000 & 0.078 \\
Intercept & $6.878 \mathrm{E7}$ & 1 & $6.878 E 7$ & 236.21 & .000 & 0.39 \\
Gravidity & 1832523.0 & 1 & 1832523.0 & 6.29 & .013 & .49 \\
Placental malaria & 420890.8 & 2 & 210445.4 & 0.72 & .058 & 0.017 \\
Age & 1055645.5 & 1 & 1055645.5 & 3.63 & .034 & 0.010 \\
Gravidity* age & 1314216.3 & 1 & 1314216.3 & 4.51 & .036 \\
Gravidity* placental malaria & 1957844.9 & 2 & 978922.4 & 3.36 & & 0.012 \\
Error & $1.074 \mathrm{E} 8$ & 369 & 291178.3 & & & \\
Total & $3.858 \mathrm{E} 9$ & 377 & & & & \\
Corrected total & $1.165 \mathrm{E} 8$ & 376 & & & & \\
\hline
\end{tabular}

${ }^{\mathrm{a}} \mathrm{R}$-Squared $=0.078$ (Adjusted R-Squared $=0.060$ ).

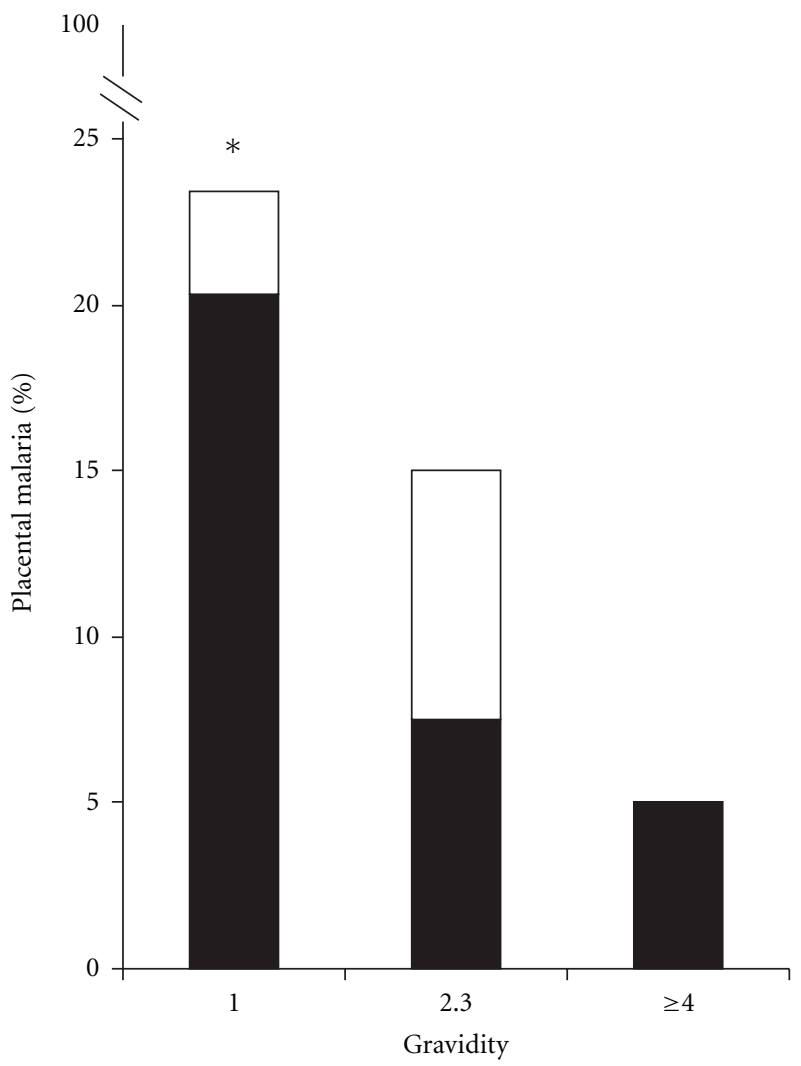

$\square$ Past

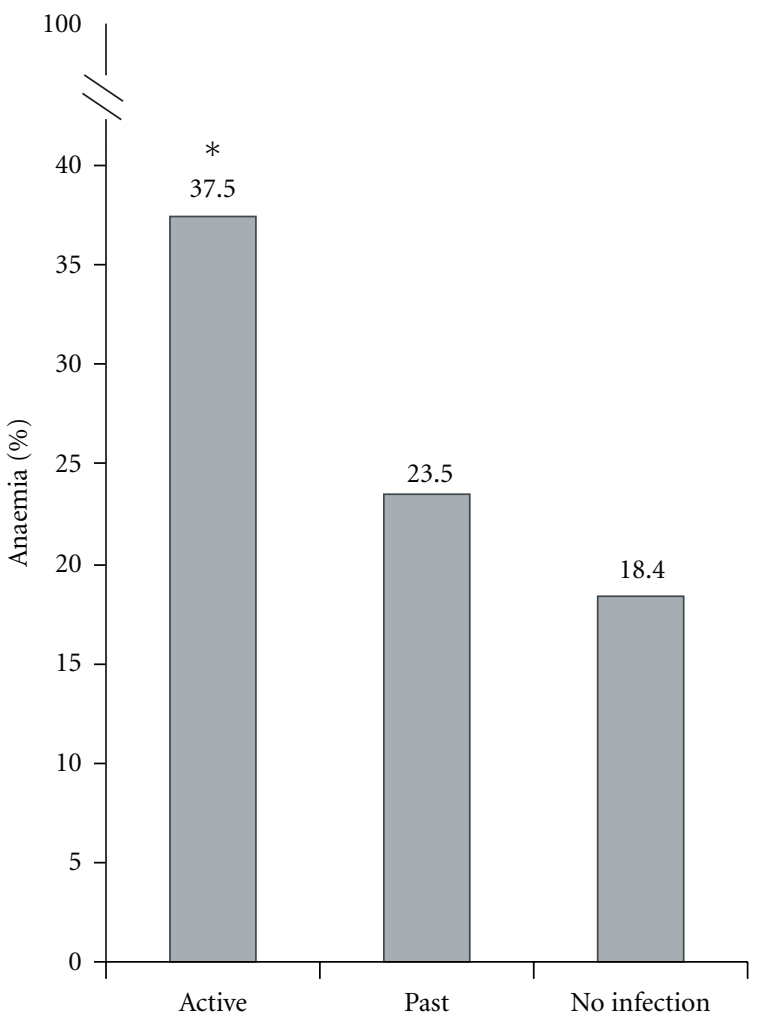

(a)

(b)

Figure 1: (a) Prevalence of placental malaria by gravidity. ${ }^{*} P<.05$ for crude comparison between primigravidae and multigravidae. (b) Prevalence of anaemia by placental malaria infection status: active, past, or no infection. ${ }^{*} P<.005$ for comparison between active versus no infection groups.

an antigenically and functionally distinct subpopulation of pRBCs, which is clonally expanded owing to the new niche of growth provided by the placenta $[19,20]$. The functional distinction between pregnancy-associated parasites and parasites of nonpregnant individuals has partly been explained by the CSA-adhesion ability of the former $[21,22]$.
Accordingly, in a related study [23], we found CSA-adhesion to constitute a prominent functional feature of Ugandan placental pRBCs. The parity dependency, found herein, was however not as marked as previously reported from hightransmission areas [24]. This shift may be a reflection of the lower transmission level in the area but may also reflect 
TABLE 7: Effect of gravidity and placental malaria infection on mean birthweight.

\begin{tabular}{lcccc}
\hline & Placental malaria & Mean birthweight & Std. error & $(95 \%$ CI $)$ \\
\hline \multirow{3}{*}{ Primigravidae } & Active infection & 3015 & 105.8 & $(2810-3220)$ \\
& Past infection & 3180 & 207.5 & $(2775-3590)$ \\
& No infection & 3180 & 65.7 & $(3050-3310)$ \\
\hline \multirow{3}{*}{ Multigravidae } & Active infection & 3440 & 114.0 & $(3220-3665)$ \\
& Past infection & 2930 & 168.9 & $(2600-3265)$ \\
\hline
\end{tabular}

The table depicts the marginal mean birthweights estimated from the following model: (Intercept) gravidity, placental malaria, age, gravidity*age, gravidity* placental malaria. Covariates appearing in the model are fixed at the following values: age $=21.91$.

TABLE 8: Pairwise comparisons of birthweight means across gravidity versus placental malaria subgroups.

\begin{tabular}{|c|c|c|c|c|c|c|c|}
\hline & & & & & & \multicolumn{2}{|c|}{$95 \% \mathrm{CI}$} \\
\hline \multicolumn{2}{|c|}{ Pair 1 versus Pair 2} & Difference in mean birthweight & Std. error & df & $P$-value & Lower & Upper \\
\hline \multirow{2}{*}{ G1* active } & $\geq \mathrm{G} 2 *$ active & -427.6 & 155.5 & 1 & .006 & -732.5 & -122.8 \\
\hline & $\geq \mathrm{G} 2 *$ no infection & -247.6 & 116.2 & 1 & .033 & -475.3 & -19.9 \\
\hline G1* no infection & $\geq \mathrm{G} 2 *$ active & -265.3 & 131.5 & 1 & .044 & -523.1 & -7.5 \\
\hline$\geq \mathrm{G} 2 *$ active & $\geq \mathrm{G} 2 *$ past & 508.1 & 204.2 & 1 & .013 & 107.9 & 908.3 \\
\hline$\geq \mathrm{G} 2 *$ past & $\geq \mathrm{G} 2 *$ no infection & -328.0 & 176.0 & 1 & .062 & -673.1 & 17.0 \\
\hline
\end{tabular}

Pairwise comparison of estimated birthweight marginal means. The mean difference is significant at the 0.05 level. Comparisons were made for all possible subgroup combinations but only significant combinations are included in the table. $\mathrm{G} 1=$ primigravidae; $\geq \mathrm{G} 2=$ multigravidae.

the presence of other confounding factors such as HIV. Interestingly, placental parasites from this region were found to interact with several placental receptors [23]; whereas exclusive CSA adhesion has been reported from highly endemic areas. Of note, parity dependency of placental infection was only significant in the crude analysis. Although a trend of higher infection rate in younger primigravidae was observed, the effect of gravidity could not be separated from age. The analysis was hampered by sample size limitations, in particular the absence of higher parities $(\geq G 4)$ in the younger age group.

Anaemia, one of the main adverse outcomes of placental infection, was present in $22 \%$ of the study participants. The women were, however, mainly affected by moderate level of anaemia. A median $\mathrm{Hb}$ level of $12.3 \mathrm{~g} / \mathrm{dl}$ and the low prevalence of severe anaemia (0.8\%) may reflect a good general health status but is also comparable with levels reported previously from low- and intermediate-transmission areas in Africa [4]. A majority of the study participants had received iron and folic acid supplementation during pregnancy (79.3\% and $70.4 \%$, respectively; Table 1$)$, which may in part explain the moderate level of anaemia observed in the study population.

Active placental infection constituted the only significant risk factor for anaemia in women of all parities $(\mathrm{OR}=$ 2.8). In the tropics, anaemia is multifactorial and may be caused by iron deficiency, parasitic infections, and haemoglobinopathies. Available data also suggest that severe anaemia is more common in women coinfected by malaria and HIV, which probably is a consequence of the increased level of parasitaemia observed in HIV-infected pregnant women $[25,26]$. The potential role of infections other than malaria was not assessed in this study. In a recent study from Western Uganda, both hookworm infections and malaria were reported to be considerably associated with anaemia during pregnancy [27].

Whilst stillbirths and preterm deliveries were uncommon at Mulago's labour suite, delivery of LBW babies was more prevalent $(12.2 \%)$. No direct associations were found between placental infection and LBW or mean birthweight (Tables 5 and 6). Parity and placental infection were, however, found to have an interactive effect on mean birthweight $(P=.036)$. Birthweight reduction was most obvious in primigravidae with active infection and multigravidae with past infection (Tables 7 and 8). It is however difficult to assess the significance of the observed pattern as the role of other confounding factors could not be assessed due to sample size limitation.

The worst birthweight outcomes, for example, LBW, $(<2500 \mathrm{~g})$ have previously mainly been reported to be associated with chronic infections $[28,29]$. A recent longitudinal study has also reported that the risk of LBW is higher with increased frequency of malaria episodes and with infections occurring in the second than in the third trimester or at delivery [30]. The majority of the cases in our study was afflicted by acute infection suggesting that infection was contracted close to delivery, which may explain the lack of association between placental infection and LBW. Moreover, 
the low transmission level in the area in combination with better access of preventive measures in urban settings probably decreases the number of malaria episodes and prevents the establishment of stubborn chronic infections, hence affecting the risk of LBW.

Increased haemoglobin level was interestingly identified as the only factor with a strong protective effect against LBW $(\mathrm{OR}=0.83$; Table 5$)$. According to previous reports, maternal anaemia may on its own, independent of placental malaria infection, contribute to intrauterine growth retardation and consequently LBW, most likely via a reduction in oxygen transport to the foetus [13].

Antenatal clinics constitute an important channel for administration of IPT and for passing on information on malaria prevention in general. A great majority of the women in this study attended ANC during their pregnancy (96.8\%), which is comparable to the national levels (ANC attendance $=94 \%$ ) reported in Uganda Demographic Health Survey of year 2000-2001. Still, relative to this, the coverage of IPT was low $(41.5 \%)$, with a majority receiving only one dose of SP. Uganda's national policy for IPT administration during pregnancy is 2 doses of SP. Of note, significantly fewer primigravidae used IPT as compared to multigravidae (Table 2; G1 $=31.6 \%$; G2-3 = 49.3\%; $\geq \mathrm{G} 4=55.7 \%$ ). The low coverage may be explained by inadequate knowledge on how to offer IPT by the health workers and/or scarcity of the drugs. It is pivotal to reach first-time mothers in time, especially since they are more prone to the most adverse outcomes of PAM. The use of bednet was widespread (67.3\%) but only $32 \%$ were strict ITN users, a number that is unacceptably low. Using bednet significantly protected against placental malaria $(\mathrm{OR}=0.56$; Table 3$)$. A trend of protective effect, although not significant, was also observed with anaemia. Considering their ease of use and reported benefits to both mother and the newborn baby [31], increased accessibility and use of low-cost ITNs should be more promoted.

This study was limited by the small sample size, the lack of HIV, and helminth infection data, which may have weakened observed associations. The HIV seroprevalence in pregnant women at Mulago has been around 11\% during the past recent years, a level that is higher than that of the national estimates $(6.4 \%)$ but comparable to the estimates in other major urban hospitals in Uganda. Hence, although we excluded AIDS patients, a proportion of HIV-positive mothers are most probably present in the study population. Moreover, the study may be biased by the time of patient recruitment which was limited to weekdays between 8:00. a.m and 5:00 p.m. Despite these limitations, our study provides a first glimpse of the burden of malaria in pregnancy at Uganda's National Referral Hospital in an urban area of lower transmission. It may also have bearings for the future design of larger studies and the development of public health policies to prevent PAM, maternal anaemia, and LBW.

Ultimately, to gain a better insight on the actual clinical burden of malaria in pregnancy versus transmission level and to identify additional high-risk subpopulations, data from multi-site longitudinal cohort studies are required. However, such studies are logistically demanding, thus very infrequent but would, for example, provide insights on disease kinetics and enable the optimal targeting of preventive interventions, such as IPT, during pregnancy.

\section{Authors' Contributions}

F. Namusoke was in charge of patient enrollment, management and care, specimen collection and helped in data analysis and manuscript writing. N. Rasti co-supervised laboratory-related activities, analyzed the data, drafted and revised the manuscript and assisted in manuscript submission. F. Kironde co-supervised laboratory-related activities, helped in data analysis and manuscript writing. M. Wahlgren helped in manuscript writing and coordinated manuscript submission. F. Mirembe was in charge of hospital-related activities and helped in manuscript writing. All authors conceptualized and designed the study together, read and approved the final version of the manuscript.

\section{Acknowledgments}

The authors are grateful to the pregnant women who participated in this study. They also thank the laboratory/hospital staff at the Labour Unit, the Department of Pathology (special praise goes to Dr. H. Wabinga) and the members of the Department of Biochemistry at Mulago Hospital for their technical assistance. Special thanks to Dr. S. Balyejjusa, S. Nanyonga, P. Kakeeto, and J. P Mpindi. This work was supported by grants from BioMalPar (LSHPCT-2004-503578), the Swedish International Development Agency (SIDA-SAREC), the Swedish Research Council, and the Foundation of Erik \& Edith Fernström. F. Namusoke and N. Rasti contributed equally to this work.

\section{References}

[1] B. J. Brabin, "An analysis of malaria in pregnancy in Africa," Bulletin of the World Health Organization, vol. 61, no. 6, pp. 1005-1016, 1983.

[2] H. L. Guyatt and R. W. Snow, "Impact of malaria during pregnancy on low birth weight in sub-Saharan Africa," Clinical Microbiology Reviews, vol. 17, no. 4, pp. 760-769, 2004.

[3] R. W. Steketee, B. L. Nahlen, M. E. Parise, and C. Menendez, "The burden of malaria in pregnancy in malaria-endemic areas," American Journal of Tropical Medicine and Hygiene, vol. 64, no. 1-2, pp. 28-35, 2001.

[4] H. L. Guyatt and R. W. Snow, "The epidemiology and burden of Plasmodium Falciparum-related anemia among pregnant women in sub-Saharan Africa," American Journal of Tropical Medicine and Hygiene, vol. 64, no. 1-2, pp. 36-44, 2001.

[5] T. Leenstra, P. A. Phillips-Howard, S. K. Kariuki et al., "Permethrin-treated bed nets in the prevention of malaria and anemia in adolescent schoolgirls in Western Kenya," American Journal of Tropical Medicine and Hygiene, vol. 68, no. 4, pp. 86-93, 2003.

[6] C. Menendez, J. Ordi, M. R. Ismail et al., "The impact of placental malaria on gestational age and birth weight," Journal of Infectious Diseases, vol. 181, no. 5, pp. 1740-1745, 2000 . 
[7] S. J. Rogerson, N. R. Van den Broek, E. Chaluluka, C. Qongwane, C. G. Mhango, and M. E. Molyneux, "Malaria and anemia in antenatal women in Blantyre, Malawi: a twelve-months survey," American Journal of Tropical Medicine and Hygiene, vol. 62, no. 3, pp. 335-340, 2000.

[8] A. Walker-Abbey, R. R. T. Djokam, A. Eno et al., "Malaria in pregnant Cameroonian women: the effect of age and gravidity on submicroscopic and mixed-species infections and multiple parasite genotypes," American Journal of Tropical Medicine and Hygiene, vol. 72, no. 3, pp. 229-235, 2005.

[9] F. Nosten, F. ter Kuile, L. Maelankirri, B. Decludt, and N. J. White, "Malaria during pregnancy in an area of unstable endemicity," Transactions of the Royal Society of Tropical Medicine and Hygiene, vol. 85, no. 4, pp. 424-429, 1991.

[10] H. L. Guyatt and R. W. Snow, "Malaria in pregnancy as an indirect cause of infant mortality in sub-Saharan Africa," Transactions of the Royal Society of Tropical Medicine and Hygiene, vol. 95, no. 6, pp. 569-576, 2001.

[11] S. C. Murphy and J. G. Breman, "GAPS in the childhood malaria burden in Africa: cerebral malaria, neurological sequelae, anemia, respiratory distress, hypoglycemia, and complications of pregnancy," American Journal of Tropical Medicine and Hygiene, vol. 64, no. 1-2, pp. 57-67, 2001.

[12] M. C. Nunes and A. Scherf, "Plasmodium falciparum during pregnancy: a puzzling parasite tissue adhesion tropism," Parasitology, vol. 134, no. 13, pp. 1863-1869, 2007.

[13] M. R. Ismail, J. Ordi, C. Menendez et al., "Placental pathology in malaria: a histological, immunohistochemical, and quantitative study," Human Pathology, vol. 31, no. 1, pp. 85-93, 2000.

[14] F. H. Verhoeff, B. J. Brabin, S. Van Buuren et al., "An analysis of intra-uterine growth retardation in rural Malawi," European Journal of Clinical Nutrition, vol. 55, no. 8, pp. 682-689, 2001.

[15] A. Yeka, K. Banek, N. Bakyaita et al., "Artemisinin versus nonartemisinin combination therapy for uncomplicated malaria: randomized clinical trials from four sites in Uganda," PLoS Medicine, vol. 2, no. 7, article e190, 2005.

[16] J. N. Bulmer, F. N. Rasheed, N. Francis, L. Morrison, and B. M. Greenwood, "Placental malaria. I. Pathological classification," Histopathology, vol. 22, no. 3, pp. 211-218, 1993.

[17] J. N. Bulmer, F. N. Rasheed, L. Morrison, N. Francis, and B. M. Greenwood, "Placental malaria. II. A semi-quantitative investigation of the pathological features," Histopathology, vol. 22, no. 3, pp. 219-225, 1993.

[18] S. J. Rogerson, P. Mkundika, and M. K. Kanjalal, "Diagnosis of Plasmodium falciparum malaria at delivery: comparison of blood film preparation methods and of blood films with histology," Journal of Clinical Microbiology, vol. 41, no. 4, pp. 1370-1374, 2003.

[19] M. Fried, F. Nosten, A. Brockman, B. J. Brabin, and P. E. Duffy, "Maternal antibodies block malaria," Nature, vol. 395, no. 6705, pp. 851-850, 1998.

[20] C. H. Ricke, T. Staalsoe, K. Koram et al., "Plasma antibodies from malaria-exposed pregnant women recognize variant surface antigens on Plasmodium falciparum-infected erythrocytes in a parity-dependent manner and block parasite adhesion to chondroitin sulfate A," Journal of Immunology, vol. 165, no. 6, pp. 3309-3316, 2000.

[21] J. G. Beeson, G. V. Brown, M. E. Molyneux, C. Mhango, F. Dzinjalamala, and S. J. Rogerson, "Plasmodium falciparum isolates from infected pregnant women and children are associated with distinct adhesive and antigenic properties," Journal of Infectious Diseases, vol. 180, no. 2, pp. 464-472, 1999.
[22] M. Fried and P. E. Duffy, "Adherence of Plasmodium falciparum to chondroitin sulfate A in the human placenta," Science, vol. 272, no. 5267, pp. 1502-1504, 1996.

[23] N. Rasti, F. Namusoke, A. Chêne et al., "Nonimmune immunoglobulin binding and multiple adhesion characterize Plasmodium falciparum-infected erythrocytes of placental origin," Proceedings of the National Academy of Sciences of the United States of America, vol. 103, no. 37, pp. 13795-13800, 2006.

[24] M. Desai, F. O. ter Kuile, F. Nosten et al., "Epidemiology and burden of malaria in pregnancy," Lancet Infectious Diseases, vol. 7, no. 2, pp. 93-104, 2007.

[25] R. W. Steketee, J. J. Wirima, L. Slutsker et al., "Malaria parasite infection during pregnancy and at delivery in mother, placenta, and newborn: efficacy of chloroquine and mefloquine in Rural Malawi," American Journal of Tropical Medicine and Hygiene, vol. 55, no. 1, pp. 24-32, 1996.

[26] F. H. Verhoeff, B. J. Brabin, C. A. Hart, L. Chimsuku, P. Kazembe, and R. L. Broadhead, "Increased prevalence of malaria in HIV-infected pregnant women and its implications for malaria control," Tropical Medicine and International Health, vol. 4, no. 1, pp. 5-12, 1999.

[27] R. Ndyomugyenyi, N. Kabatereine, A. Olsen, and P. Magnussen, "Malaria and hookworm infections in relation to haemoglobin and serum ferritin levels in pregnancy in Masindi district, western Uganda," Transactions of the Royal Society of Tropical Medicine and Hygiene, vol. 102, no. 2, pp. 130-136, 2008.

[28] B. J. Brabin, C. Romagosa, S. Abdelgalil et al., "The sick placenta-the role of malaria," Placenta, vol. 25, no. 5, pp. 359-378, 2004.

[29] C. E. Shulman, T. Marshall, E. K. Dorman et al., "Malaria in pregnancy: adverse effects on haemoglobin levels and birthweight in primigravidae and multigravidae," Tropical Medicine and International Health, vol. 6, no. 10, pp. 770-778, 2001.

[30] L. Kalilani, I. Mofolo, M. Chaponda, S. J. Rogerson, and S. R. Meshnick, "The effect of timing and frequency of Plasmodium falciparum infection during pregnancy on the risk of low birth weight and maternal anemia," Transactions of the Royal Society of Tropical Medicine and Hygiene, vol. 104, no. 6, pp. 416-422, 2010.

[31] C. Gamble, J. P. Ekwaru, and F. O. ter Kuile, "Insecticidetreated nets for preventing malaria in pregnancy," Cochrane Database of Systematic Reviews, no. 2, Article ID CD003755, 2006. 


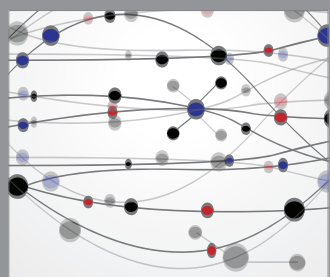

The Scientific World Journal
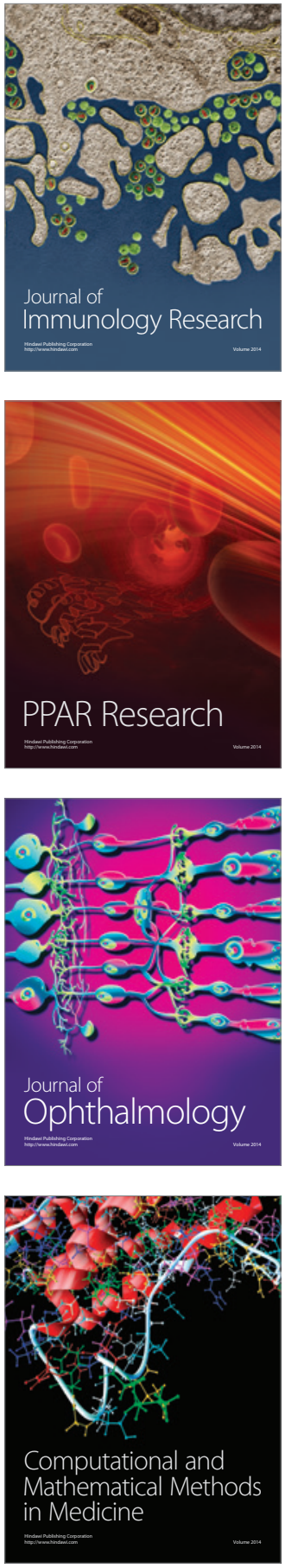

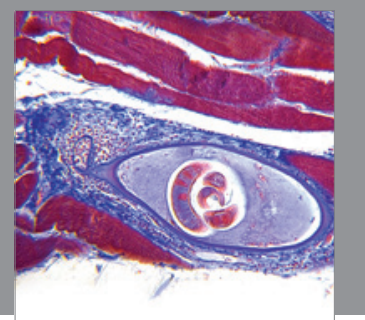

Gastroenterology

Research and Practice
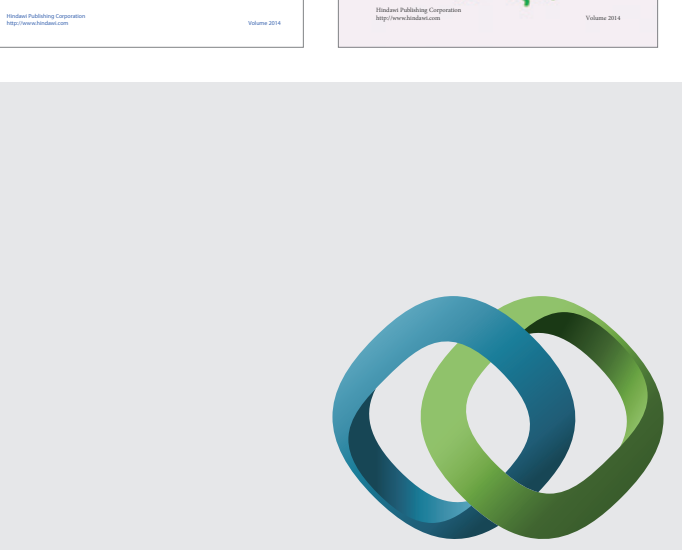

\section{Hindawi}

Submit your manuscripts at

http://www.hindawi.com
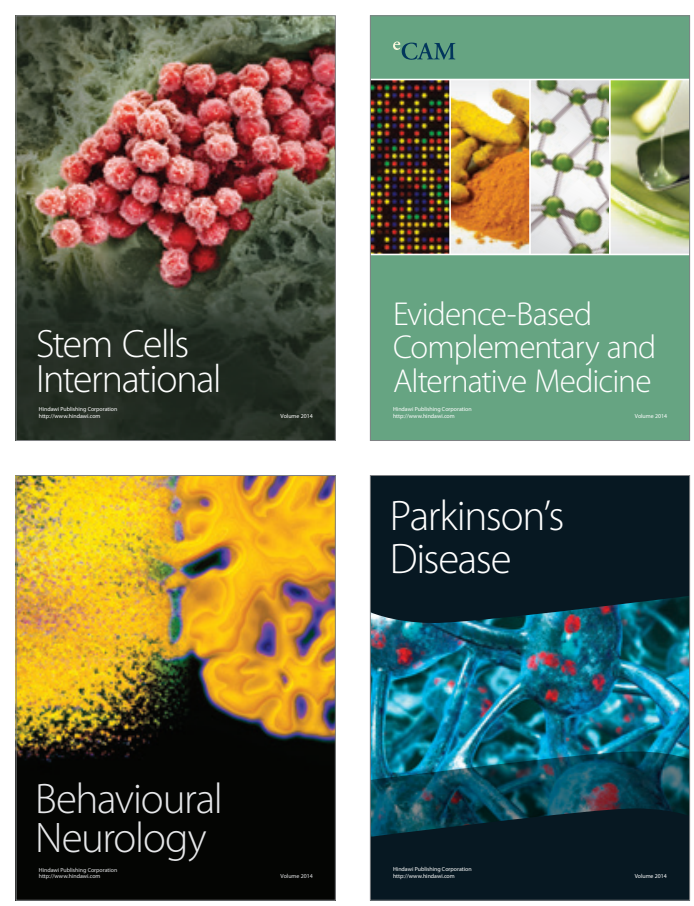

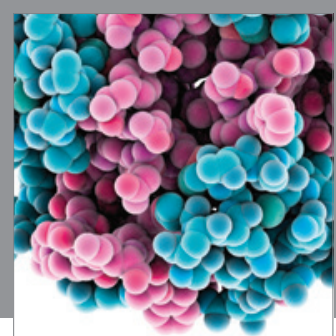

Journal of
Diabetes Research

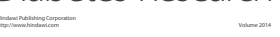

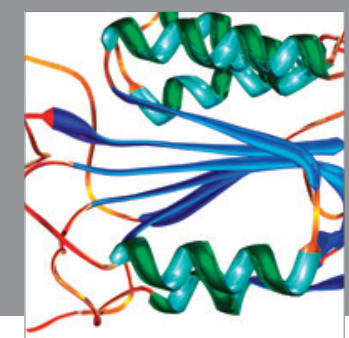

Disease Markers
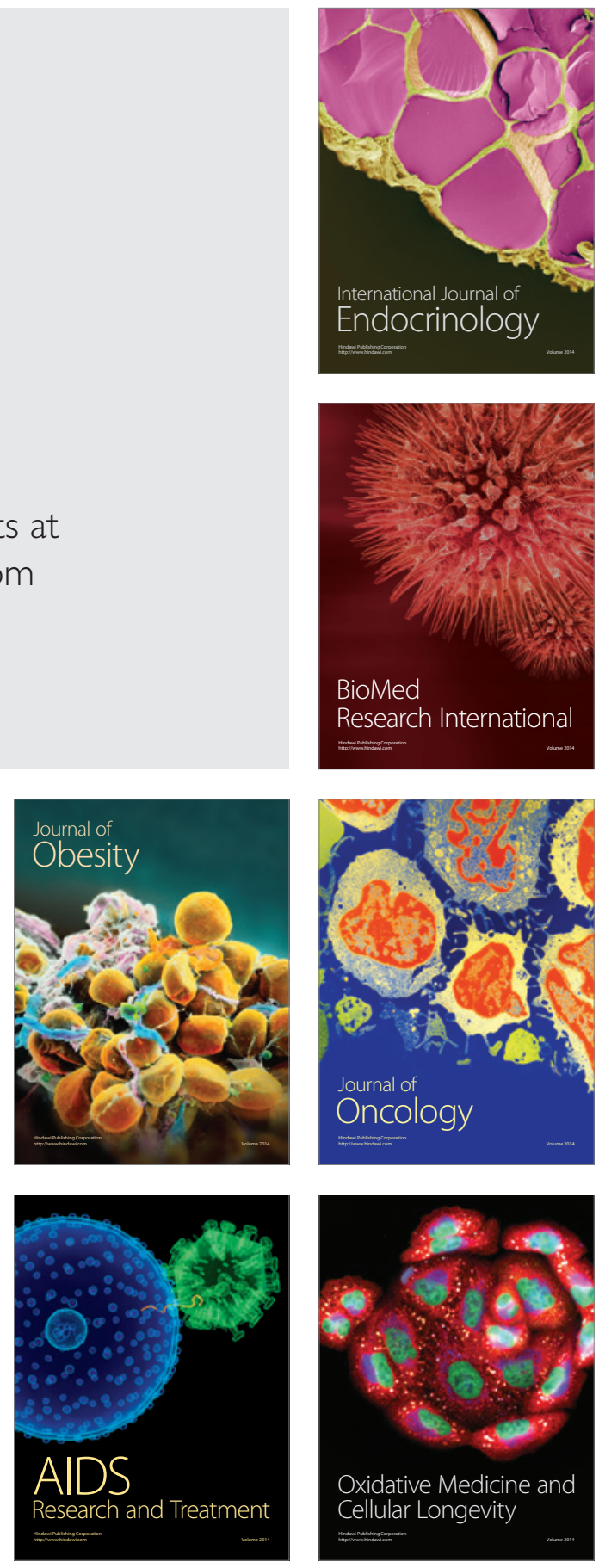